\title{
Análisis comparativo regional del desarrollo de la administración local española en internet (1997-2002)
}

\author{
Celia Chaín Navarro \\ Departamento de Información y Documentación. \\ Universidad de Murcia \\ E-mail:chain@um.es \\ Antonio Muñoz Cañavate \\ Área de Biblioteconomía y Documentación. \\ Universidad de Extremadura \\ E-mail: amunoz@alcazaba.unex.es
}

\section{RESUMEN}

El trabajo presenta el resultado de un proyecto de investigación llevado a cabo en España de 1997 a 2002, y que ha registrado la presencia en Internet de una de las tres Administraciones Públicas existentes, en este caso la Administración local, que es a su vez la más cercana al ciudadano. Se pretende conocer la evolución de las corporaciones locales estructuradas en el contexto geográfico regional; averiguar si la variable dominio propio/ajeno sirve como parámetro de medida sobre el desarrollo de los sistemas web locales, y comprobar la aplicabilidad del método de las confrontaciones 4 a 4 , que consiste en agregar las cuatros regiones que tienen mayor PIB, y las cuatro que tienen menos, y comparar el desarrollo económico y el del sitio web. Se confirma la evolución constante y heterogénea de las web locales, y se validan los otros dos métodos.

Palabras clave: Información al ciudadano; Administración local; Análisis regional; Ayuntamientos; Internet; Cibergobierno; Administración electrónica; wWw; Políticas de información, Ciberdemocracia; Dominios.

\section{COMPARATIVE REGIONAL ANALYSIS OF THE DEVELOPMENT OF SPANISH LOCAL ADMINISTRATION IN INTERNET (1997-2002)}

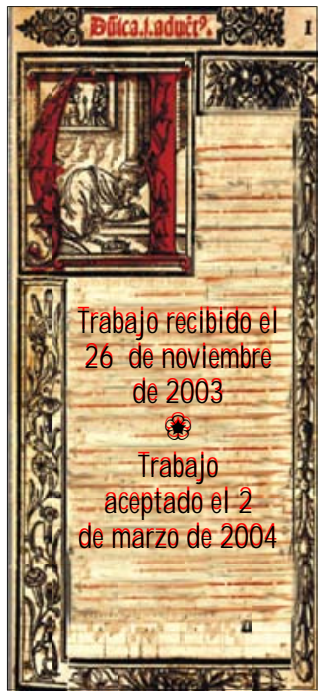

Celia Chaín-Navarro y Antonio Muñoz-Cañavate

\begin{abstract}
The paper is the result of a research project carried out in Spain between 1997 and 2002, in which the presence in Internet of one of three existing Public Administrations was recorded, specifically local government, which is the closest to the public. We wanted to learn about the local corporate structures within their regional geographical context, and to find out whether the variable own/outside-domain could be taken as measure of the development of local websites. We also intended to find
\end{abstract}


out whether the 4 to 4 confrontation method (which consists of adding the 4 regions with the highest GNP, and the 4 with the lowest) could be applied to compare economic development with that of the website. The permanent and heterogeneous evolution of local websites is confirmed, and the two other methods are validated.

Keywords: Information for Citizens; Local Administration; Internet; E-Government; e-Administration; Www; Information Politics.

\section{INTRODUCCIÓN}

C on la llegada de Internet al servicio de las distintas Administraciones Públicas aparece un nuevo campo de estudio que, bajo la denominación de eGoremment o Administración electrónica, analiza una nueva realidad al servicio del ciudadano.

Las administraciones Públicas encuentran una nueva forma de actuación con los administrados, que permite redefinir los sistemas de información que soportan en sus distintos flujos, ya sean de entrada, internos o de salida. Así, los procesos de salida, aquéllos que interactúan con agentes externos, pueden ser estudiados desde distintos puntos de vista: como la presencia en la red; los recursos de información y los servicios que distribuyen; los procesos denominados de democracia electrónica, que permiten abrir el debate de los asuntos públicos a los ciudadanos a través de los foros electrónicos; o simplemente el estudio del Weben el contexto general de las políticas deinformación.

Precisamente, Alfons Cornella ${ }^{1}$ diferenciaba en 1996, en el marco de las políticas deinformación, cuatro tipo de acciones que son llevadas a cabo por los poderes legislativo y ejecutivo, y que se distinguen a través de:

* acciones legislativas o reguladoras;

* desarrollo de infraestructuras informacionales;

- desarrollo de infoestructuras;

* y mejora de la gestión de información en la administración pública.

Dos años más tarde, en otro de sus trabajos, Cornella ${ }^{2}$ estudió las políticas de información en España en tres direcciones: legislación, acciones de promoción y estímulo, y gestión de lainformación en las administraciones públicas.

1 Alfons Cornella. "Políticas de información y rankings de países", en El profesianal delainfomación, julio-agosto 1996, vol. 7, n. 7-8, p. 24.

2 Alfons Cornella. "Information Policies in Spain", en GouemmentInfomationQuartely, 1998, vol. 15, n? 2, p. 197-200. 


\section{Investigacón Biblicteedógica v. 18 No. 36 enero/ junio de 2004}

El LibroV edesdbrelainfomacióndd setarpúblicoenlasociedaddelainformacón publicado en $1998{ }^{3}$ por la Unión Europea, estructura los procesos entre Administración y administrados en tres dimensiones:

a) servicios de información,

b) servicios de comunicación,

c) servicios de transacción.

A su vez, cada uno de ellos aplicados a tres vertientes:

a) vida cotidiana,

b) administración a distancia,

a) y participación política.

Un estudio 4 elaborado para la Unión Europea en 2002 establece cuatro grupos de usuarios en la relación que se establece conla administración pública:

* Administración-ciudadano,

* Administración-empresa,

* Administración-administración,

* Administración-empleados de la administración.

Otro informe ${ }^{5}$ de la UE del mismo año, establece en cuatro fases el grado de desarrollo de los servicios dela administración electrónica:

* Fase 1. Información: información en línea sobre el servicio público.

* Fase 2. Interacción: descarga en línea de formularios.

* Fase 3. Interacción bilateral: cumplimentación y envío de formularios, acceso a bases de datos, etcétera..

* Fase 4. Transacción: permite la formalización y entrega con acuse de recibo de los formularios, incluido el pago del servicio si procede.

Traspasando fronteras continentales, fueron los Estados Unidos de América los que inciaron las investigaciones sobre la inclusión de la administración pública en Internet a mitad delos años noventa. Stowers hace un repaso de la bibliografía existente

3 Unión Europea. Lainformación dd setor pública un rearsodavepara Eurqpa COM (1998), 585.

4 Unión Europea. Infomesdarela edduaón delos seviaios públicos deetrónicos dentrodela UE [en línea]. 25-26 abril 2002. Disponible en http://www.csic.map.es/csi/pdf/eGovEsp_definitivo.pdf [Fecha de consulta 28-10-2003].

5 Gloria Nistal y José M. Castro. RacionalizacoóndelaspdíticasdeAdministracoóndectrónicaenlaUniónEut ropea [en línea] (Estudio encargado por la Presidencia Española del Consejo de la Unión Europea). 2002. Disponible en http:// www.csi.map.es/csi/pdf/RacionalEsp.pdf [fecha de consulta: 28 octubre 2003]. 
hasta el momento. Dice que en 1999 los estudios estaban empezando: eran casi anecdóticos ${ }^{6}$ o encuestas. ${ }^{7}$ Se centraban en cómo gobernar, ${ }^{8}$ particularizaban en la legislación estatal ${ }^{9}$, y en cómo los ciudadanos usan la tecnología ${ }^{10} \mathrm{o}$ la potencialidad de la tecnología para favorecer el pluralismo ${ }^{11}$ y sobre el discurso político. ${ }^{12}$ Muchos se han centrado en estudiar las características demográficas de la población que accede a Internet y sobre discusiones de la política cultural que envuelve Internety la web. ${ }^{13}$

En los últimos años del siglo XX y los primeros del siguiente, cuando la "e-inclusión" de la administración pública era ya notoria tanto en Europa como en América, se han empezado a proponer modelos, fases y etapas por las que debían pasar las administraciones hasta imbuirse de lleno en la etapa de administración electrónica. El de McClure, que fue uno de los pioneros, ofreció en 1997 un estudio detallado sobre cómo evaluar los recursos y servicios de información de las webs estatales, ${ }^{14}$ al que

6 D. Schooley. Local Government Go on the WWW. En FonumforPdighRerchnens Camden: State University of New Jersey, 1996. p. 1-3 y 15; MOURA, B. San Carlos Discovers the Internet and Shares Lessons That Were Learned. PublicManagment, 1996, p. 31-37; P. Greenberg. On the Net: Political Possibilities. StateLegjatures 1996. p. 19-23; COUNCIL of Sate Government. The Cyber Compaigns. SateGouemmentNenş 1995, p. 29-31; R.Gutwitt, Innovation by Wire. Goueming 1994, p. 52-57.

7 B. Bimber, TheDengraphics of Intemet Acess [en línea]. http://www.sscf.ucsb.edu/ survey1/access.html. [1997] (No disponible actualmente en Internet); B. Bimber, TheIntemet and Pditical

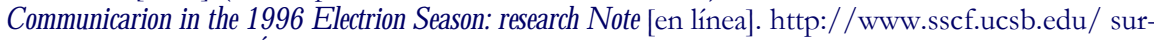
vey1/mobilize.htm [Última consulta 1997] (No disponible actualmente en Internet); B. Bimber, TheIntemetandPditical Transfomation[en línea]. http://www.sscf.ucsb.edu/ survey1/poltran2.htm [Última consulta 1996] (No disponible actualmente en Internet) y B. Fisher, M. Margolis \& D. Resnick. Breaking Ground on the Virtual Frontier Surveying Civic Life on the Internet. TheAmarican Soidogist, 1996, p. 11-29.

8 P. Greenberg. On the Net: Political Possibilities... 1996; B. Moura, San Carlos Discovers the Internet and Shares Lessons That Were Learned... 1996, y D. Schooley. Local Government Go on the WWW... 1996; COUNCIL of Sate Government. The Cyber Compaigns... 1995, y R. Gutwitt. Innovation by Wire... 1994.

9 J.A. Bourquard \& P. Greenberg. Savy Citizens. Statelegislatures March, 1995. p. 29-33; C. Blanton, Zapping the Capitol. StateGouemmentNens feb, 1995, p. 16-19, y J. A. Bourquard. Legislatueres Hit Cyberspace. StateLegidatures aug. 1994, p. 31-33.

10 J. A. Bourquard \& P. Greenberg. Savy Citizens... 1995; H. Rheingold. TheVirtual CommmityHo mestandingon thedetranicFranties. Reading: Addison-Wesley, 1993.

11 B. J. Neubauer \& Ch. Commuri. Government Administration in the Internet Age Cyberspace and the Information Elite. Meting of theAmerican Pditical SaienceAssoiation 29 aug-1 sep. 1996. San Francisco.

12 B. Bimber. TheIntemetandPditical Transfomation[en línea]. [Última consulta 1996] (No disponible actualmente en Internet).

13 B. Bimber, TheIntemetandPditical Transfomation[en línea] [Última consulta 1996] (No disponible actualmente en Internet), y B. Fisher, M. Margolis \& D. Resnick. Breaking Ground on the Virtual Frontier Surveying Civic Life on the Internet... 1996.

14 C. R. McClure; S.K. Wyman \& J.C. Beachboard. QualityCriteia for EvaluatingInfomation Resarres and Services Available fram Feakal Web Sites [en línea]. Disponible en ttp://www.oclc.org/research/publications/art/1997/mcclure/app3.htm 


\section{Inestigacón Biblidtecdógica v. 18 No. 36 enero/ junio de 2004}

han seguido otros como el de Stowers citado, o el de Layne y Lee en 2001, y sus conocidas cuatro etapas en el "e-gobierno". ${ }^{15}$

Resumidamente los estudios han ido dirigiéndose paulatinamente desde el enfoque teórico clásico, pasando por los necesarios estudios principalmente cuantitativos, hastallegara una etapa en la que se proponen modelos y criterios de evaluación.

Hemos señalado con anterioridad que la aparición de estos sistemas de información en el contexto de las distintas Administraciones públicas, permite generar toda una nueva vertiente de estudios sobre políticas de información, y en este artículo proponemos otra variable de estudio para analizar esas políticas.

\section{ASPECTOSLEGISLATIVOSY POLÍTICAS DE INFORMACIÓN}

Existen dos factores de desarrollo básicos para permitir el desarrollo de políticas deinformación en un territorio.Éstas son:

a) un marco normativo y legislativo que permita clarificar cuáles son los deberes de

las administraciones y cuáles son los derechos de los ciudadanos,

b) y un catálogo de iniciativas y planes políticos que generen proyectos y realidades.

En España estos factores han tenido en la última década un importante desarrollo. Así, la Ley30/ 1992 deRégimenJuńdicodelas Administradanes Públicasyda Procedmiento AdministrativoComún estableció las bases reglamentarias, entre las que se encuentra la información al ciudadano para el conjunto de las administraciones.

Normativas posteriores han estructurado los servicios de información al ciudadano en la administración central, ${ }^{16}$ la protección de los datos de carácter personal, ${ }^{17}$ el uso de las TIC en el trato con los ciudadanos, ${ }^{18}$ los registros y notificaciones telemáticas, ${ }^{19}$ los servicios de la sociedad de la información y el comercio electrónico, ${ }^{20}$ la firma electrónica. ${ }^{21}$ Algunas de ellas hansido posteriormente adaptadas a nuevas realidades.

Numerosos han sido también los planes políticos tanto a nivel general, para el conjunto de la administración central, como en departamentos ministeriales. Así el PlandeModamizacónyMgjoradelaAdministracoónde Estadbde1992 incluía entre sus líneas estratégicas la mejora de la información y comunicación con el ciudadano. Y

15 Karen Layne \& Lee, Jungwoo. "Developing fully functional E-government: a four stages model", en Govemment Infomation Quartely, 2001, 18, p. 122-136.

16 Real Decreto 208/1996, de 9 de febrero, sobre Regulación de los Servicios de Información Administrativa y Atención al Ciudadano.

17 La Ley Orgánica 15/99 de Protección de Datos Personales.

18 Real Decreto 263/1996, de 16 de febrero, sobre Regulación de la Utilización de técnicas electrónicas, informáticas y telemáticas en la Administración.

19 Decreto 209/2003, de 21 de febrero, por el que se regulan los registros y las notificaciones telemáticas, así como la utilización de medios telemáticos para la sustitución de la aportación de certificados por los ciudadanos.

20 Ley 34/2002, de 11 de julio, de servicios de la sociedad de la información y de comercio electrónico.

21 Real Decreto-ley 14/1999, de 17 de septiembre sobre firma electrónica. 
más recientemente el PlanInfoXXI22 de 1999, sustituido en 2003 por el Plan Españą, que tendrá vigencia desde 2004 a 2005, con el objetivo de impulsar los proyectos de Sociedad de la información en España. En él se desarrollan diecinueve medidas para el impulso de la administración electrónica en un claro intento de mejorar el servicio para el ciudadano, y se mantiene la idea de ofrecer los servicios de todas las administraciones en un portal único. En la misma dirección nace la empresa pública Redes con objeto de que a través de una serie de acciones y funciones se pueda contribuir al desarrollo de la sociedad de la información en el país. ${ }^{23}$

A nivel regional son numerosas las iniciativas puestas en marcha en los últimos años, si bien no siempre con la pertinente coordinación entre las mismas, y con un avance desigual entre ellas.

\section{DIVISIÓN ADMINIST RATIVA}

El Estado español se organiza territorialmente en diecisiete Comunidades Autónomas o regiones, constituidas por más de ocho mil municipios. La agrupación de distintos municipios conforma la provincia, y ésta, a su vez, sola o con otras provincias, conforma la región. Delas diecisiete regiones, siete están formadas por una sola provincia.

Administrativamente, la gestión del Estado es responsabilidad de la Administración General del Estado. ${ }^{24}$ Las Comunidades Autónomas están regidas por un gobierno regional. ${ }^{25}$ Y los municipios por el ayuntamiento. ${ }^{26}$

22 España. Plan de acción Info XXI [en línea]. Disponible en ttp://infoxxi.es [Última consulta 06-10-2003].

23 España. Red. es [en línea]. Disponible en ttp://www.red.es [Última consulta 06-10-2003].

24 La Ley Orgánica de Funcionamiento de la Administración General del Estado, estructura y regula la Administración central en España. Son los Ministerios con toda su estructura organizativa interna (servicios centrales de los Ministerios, Secretarías y subsecretarías de Estado, Secretarías Generales Técnicas, Direcciones Generales, organismos autónomos y Entidades públicas empresariales dependientes de los Ministerios correspondientes), además de todos los servicios periféricos radicados en las provincias españolas.

25 Las Comunidades autónomas pueden formarse atendiendo a la Constitución siguiendo estas posibilidades:

a) Provincias limítrofes con características históricas, culturales y económicas comunes (Andalucía, Aragón, Cataluña, Castilla-La Mancha, Castilla-León, Comunidad Valenciana, Extremadura, Galicia, País Vasco, ...). En este grupo están las denominadas comunidades históricas (Cataluña, País Vasco y Galicia).

b) Los territorios insulares (Baleares y Canarias).

c) Las provincias que tienen entidad regional histórica por sí solas (Asturias, Cantabria, La Rioja, Madrid, Murcia y Navarra).

d) Determinados territorios cuyo ámbito no supere el de una provincia (Ceuta y Melilla, que no son comunidades, sino ciudades autónomas).

Entre las muchas competencias que les otorga la Constitución a estas comunidades está la organización de sus instituciones de autogobierno. Los Estatutos son la norma institucional de cada Comunidad, y se aprobaron en diferentes momentos según las regiones españolas, en un periodo comprendido entre 1979 y 1983.

26 Los ayuntamientos se regulan en España por la Ley de Bases de Régimen Local, y por el Reglamento de Organización, Funcionamiento y Régimen Jurídico de las Entidades Locales. 


\section{Investigaión Biblidtedlógica v. 18 No. 36 enero/ junio de 2004}

\section{OBJETIVOS}

Los grandes objetivos del estudio han sido los siguientes:

a) Conocer la presencia en Internet de cualquiera de los ayuntamientos españoles.

b) Conocer el tipo de dominio bajo el que se asientan las direcciones electrónicas de los sitios web analizados.

c) Comparar la situación entre las distintas regiones españolas, en función de la presencia de los ayuntamientos de sus municipios, y poder contrastar a unas regiones con otras en función de un indicador socioeconómico, como el PIB.

Los objetivos específicos que se marcaron en este trabajo son:

1. Comparar y contrastar esa evolución en el contexto geográfico que los engloba: las 17 comunidades autónomas españolas a las que pertenecen.

2. Comprobar la aplicabilidad de la metodología de las confrontaciones 4 a 4 comparando los porcentajes de crecimiento de los sistemas de información web locales y PIB de comunidad autónoma a la que pertenecen los ayuntamientos. Se comparan las cuatro regiones con mayor PIB con las regiones con menor PIB.

3. Conocer si la variable dominio propio sirve como parámetro de medida del desarrollo de los sistemas web locales, en las confrontaciones 4 a 4 .

\section{METOdOLOGÍA}

\section{E I sistema de dominios y su selección}

Uno de los ejes sobre los que ha gravitado el estudio global desde 1997, se centra en el conocimiento de la presencia de los ayuntamientos españoles en Internet, y el análisis de su sistema de dominios, tal y como viene marcado en los objetivos. Esto nos ha permitido definir una hipótesis de trabajo en torno al cambio del nombre de dominio, ${ }^{27}$ bajo el que se asientan las páginas Włby que permite a su vez acometer estudios sobrepolíticas de información.

Se diferencia entre sistemas wabcon dominio ajeno del tipo "http:/ / www.provee-

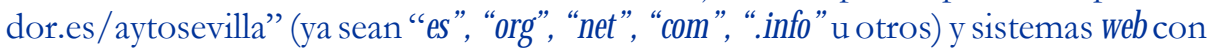
dominio propio, como en el siguiente ejemplo "http://www.ayunt-sevilla.es"(o bajo cualquiera de los dominios anteriores). La novedad de este trabajo es la asunción de lo que denominamos "dominio propio", para conocer el grado de madurez de una administración pública en Internet.

27 Según el DicainariodeIntemede Tom Fahey, el nombre de dominio es la "descripción de muchas partes conformada (de izquierda a derecha) por nombre de la computadora en la que reside la cuenta del usuario; grupo o red al cual la computadora está conectada; subdominio (por ejemplo, una universidad) y dominios principales de Internet. Un nombre de dominio se lee de lo más específico a lo más general. Los nombres de dominio se traducen a direcciones IP por medio del Sistema de Nombres de Dominio”. Tom Fahey. DicionaniodeIntemet México: Prentice-Hall, 1995. 
Se trata de asumir el hecho de que un ayuntamiento que decida incorporarse a Internet, ya está definiendo un cambio señalable en su política, aunque sea solamente porque sus responsables se han dado cuenta de que el hecho de estar en la red se convierte en un elemento de marketing que hay que tener en cuenta, o por la propia presión social de la que hablan Layney Lee (2001). ${ }^{28}$

La recolección de datos se realizaba las dos últimas semanas de cada semestre (a saber, junio y diciembre de los años 1997, 1998, 1999, 2000, 2001 y 2002) con objeto de conocer el grado de penetración de los sistemas web en la Administración local, contabilizandolos siguientes datos:

1. número total de unidades con presencia sobre las que no tienen presencia,

2. número total de unidades con dominio propio,

3. número total de unidades con dominio ajeno,

4. y, a su vez, los factores anteriores agregados por el dominio del primer nivel al que

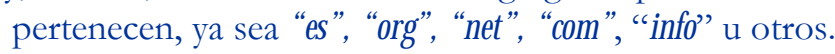

La complejidad y laboriosidad del trabajo supuso mantener una plantilla con todos los municipios divididos por provincias y, a suvez, por regiones.

Cada semestre se procedió a actualizar los datos, comprobando en primer lugar si los dominios ya registrados seguían existiendo, o si habían cambiado, y posteriormente se realizaba una búsqueda muy exhaustiva en distintos índices y catálogos, generalistas y específicos para la administración local. Además, se consultaban websites de gobiernos regionales y diputaciones provinciales, susceptibles de mantener páginas de ayuntamientos obien enlaces.

El elevado número, 8095 unidades según el Padrón de 1996, ${ }^{29}$ nos obligó a realizar una agregación total en función del número de habitantes del municipio, tras lo cual quedaron cinco intervalos (menos de 5.000 hab.; entre 5.001 y 10.000; entre 10.001 y 50.000; entre 50.001 y 100.000, ylos de más de 100.000 hab.).

Para la representación total de los datos se ha optado en este trabajo por ofrecer los resultados del conjunto del trabajo (sobre 8.095 ayuntamientos) por comunidades autónomas.

\section{L as agregaciones totales y parciales (confrontaciones cuatro a cuatro)}

Una vez obtenidos los resultados totales de los análisis efectuados se establece la necesidad de realizaranálisis sobre políticas deinformación conlos datos extraídos.

Los datos que se pueden extraer de las diferentes tablas semestrales para hacer el análisis por comunidades autónomas no siempre permitían obtener conclusiones claras. Así, se optó por utilizar un método que ya había dado buenos resultados en el

28 Karen Layne \& Lee, Jungwoo. "Developing fully functional E-government: a four stages model", en Govemment Infomation Quartely, 2001, 18, p. 122-136.

29 Web del Instituto Nacional de Estadística. http:/ / www.ine.es [última consulta 22 sept. 2003]. La variación con respecto al Padrón de 1998 es de $0.46 \%$, poco significativa. En este estudio se exceptuaron Ceuta y Melilla, al ser ciudades autónomas españolas, y no encontrarse enclavadas en ninguna provincia. 


\section{Investigacón Biblidteclógica v. 18 No. 36 enero/ junio de 2004}

estudio de las otras administraciones públicas, (precisamente la comparación de las políticas de información regionales): 30 las confrontadionesaratroaaratro Es decir, agregar datos de varias regiones, comparar con una variable socioeconómica las 4 que mayor valor tienen, y las 4 que menor valor tienen. De esta manera, las Confrontadones CuatroaCuatroutilizando como variable el PIB surgen una vez obtenidos los primeros resultados, y como una forma de comparar la evolución de los sistemas web y su relación con otros parámetros, uniendo las cuatro unidades con mayor PIB y las cuatro con menor PIB para evaluar su desarrollo en Internet.

Se ha optado por utilizar ocho para contar con una parte significativa de las diecisiete unidades objeto del estudio. Así, se trata de establecer un estudio comparativo alternativo, pero con el uso de los datos agregados cuatro a cuatro.

\section{RESULT ADOS}

La evolución general de la presencia de los ayuntamientos españoles en Internet con datos agregados aparece en la tabla1. Representa la evolución de las más de 8000 corporaciones locales agrupadas por regiones. Se puede observar claramente esa evolución, ya que desde 1997 había incluso comunidades autónomas que apenas tenían algunos ayuntamientos conectados a la red, mientras que hacia fines de 2002 había regiones que se encontraban en el $91 \%$.

En esta tabla global podemos ver cómo y cuál ha sido la evolución, la rapidez para conectarse de los ayuntamientos de algunas regiones, el salto cuantitativo que algunas han dado, ylas que más avanzaron hasta el año 2002.

Baleares lidera prácticamente el grupo durante los seis años, con un salto cuantitativo impresionante, ya que entre diciembre de 1997 y febrero de 1998, pasa de tener un $13,4 \%$ a un $77 \%$, y que a finales del periodo investigado el porcentaje es de un $91 \%$. Si volvemos a los orígenes del estudio también es importante reseñar que la comunidad autónoma que más fuerte entró en Internet, en 1997, fue Asturias, que ya tenía casi un 30\% de municipios en Internet, cifra inalcanzable para el resto, que apenas llegaban al 10\%. Sin embargo, la evolución ha sido más lenta, y en el año 1998 es Baleares la que empieza a tener las cifras mayores.

En orden numérico le sigue la Comunidad Autónoma de la Región de Murcia, con un $66,6 \%$, que desde los inicios de este estudio ha sido la segunda en porcentaje, aunque no ha dado un salto rápido sino presentado un avance continuo y más o menos homogéneo en los seis años.

30 Antonio Muñoz Cañavate \& Celia Chaín Navarro. The WoddWideWebas an information system in Spain?s Regional Administrations (1997-2000). Gouemmet InformationQuarteły, 2004 (en prensa). 
El tercer lugar lo ocupa el Principado de Asturias, con un 65,3\% y evolución semejante, y tras éstos el País Vasco (con un salto muy fuerte en el año 2000) Canarias yCataluña.

En la otra parte del ranking entre los que menos han avanzado aparecen Castilla y León, Castilla la Mancha, Aragón, La Rioja y Extremadura. Si bien muchas de estas regiones ven disminuidos sus porcentajes debido a la alta densidad de pequeños núcleos de población.

Existen diferencias importantes entre las cifras de ayuntamientos conectados a Internet en las 17 comunidades autónomas españolas, diferencias que podrían deberse a factores económicos; es decir, que las regiones más ricas podrían ser las que potencialmente se incorporaran antes a Internet, pero también podría ser determinante el número de unidades locales que tiene cada comunidad. Para comprobarlo se confeccionó la tabla2 que reúne en todas las series históricas los datos agregados de los dos grupos de regiones con los valores absolutos y los valores relativos (porcentajes) en esa comparativa 4 a 4 por PIB. Nos referimos a la unión de los datos de las cuatro regiones españolas más desarrolladas (Islas Baleares, Madrid, Cataluña y Navarra), frente a las menos desarrolladas (Murcia, Castilla la Mancha, Extremadura y Andalucía)

De la tabla2se extraen la tabla3y la figura1 (datos de todos los ayuntamientos conectados con independencia de que tengan dominios ajenos o propios), y la tabla4y la figura2(datos, sólo con los ayuntamientos que tienen dominios propios).

En ellas se puede comprobar que sí existe relación directa entre evolución web y PIB. Se puede ver que las comunidades autónomas que más producto interior bruto tienen, son aquellas que obtienen mayor porcentaje de acceso de sus corporaciones localesen Internet frente a las menos desarrolladas.

De cualquier forma, los porcentajes ayudan a ver la evolución y el desarrollo en las CC.AA de forma global, no por unidades, y ésos \% nos confirman que son las CC.AA más ricas las que tienen más ayuntamientos conectados y mayor porcentaje de dominios propios, lo que corrobora la utilidad de este tipo de confrontaciones 4 a 4 . También nos sirve para detectar que sí está muy claro el desarrollo desigual de unos y de otros, que también es evidente que las diferencias entre las regiones más ricas y las más pobres va disminuyendo en estos seis años, y que se notan menos las diferencias en cuanto a la adquisición de dominios propios por parte de los ayuntamientos de las CC.AA. más pobres, que pasan de tener una diferencia de 1.44\%/0.09\% en 1997 a $22.71 \% / 14.68 \%$. 


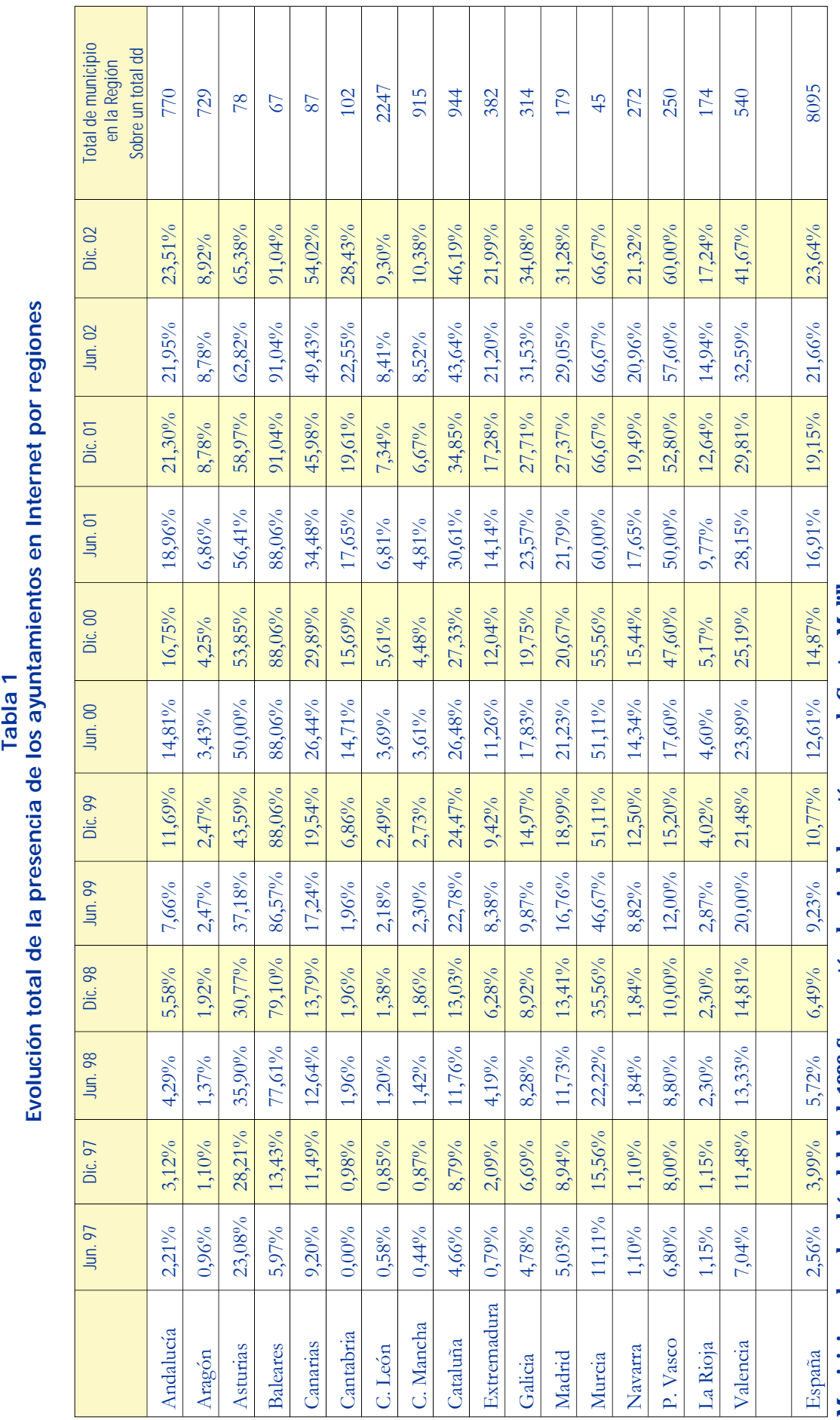




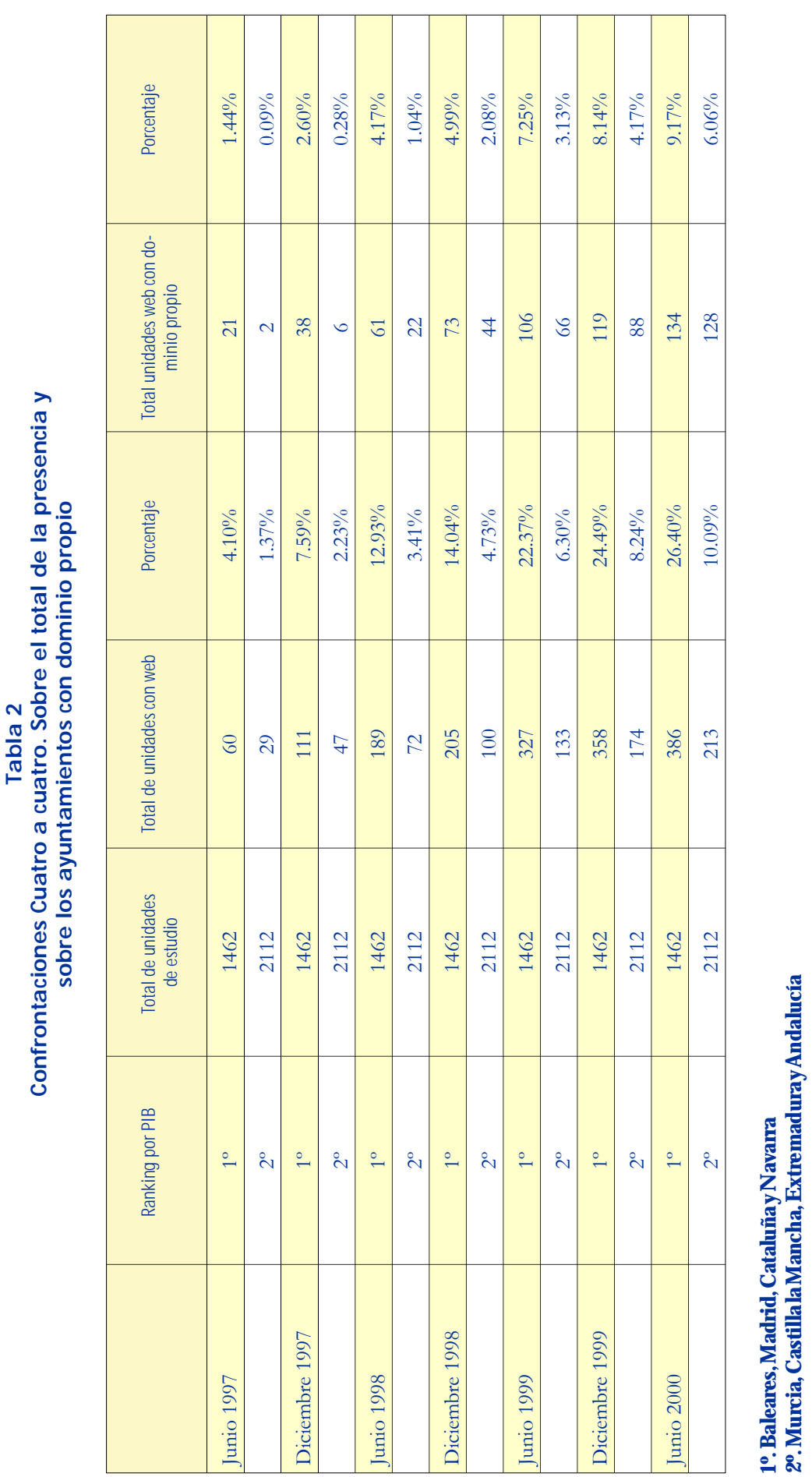




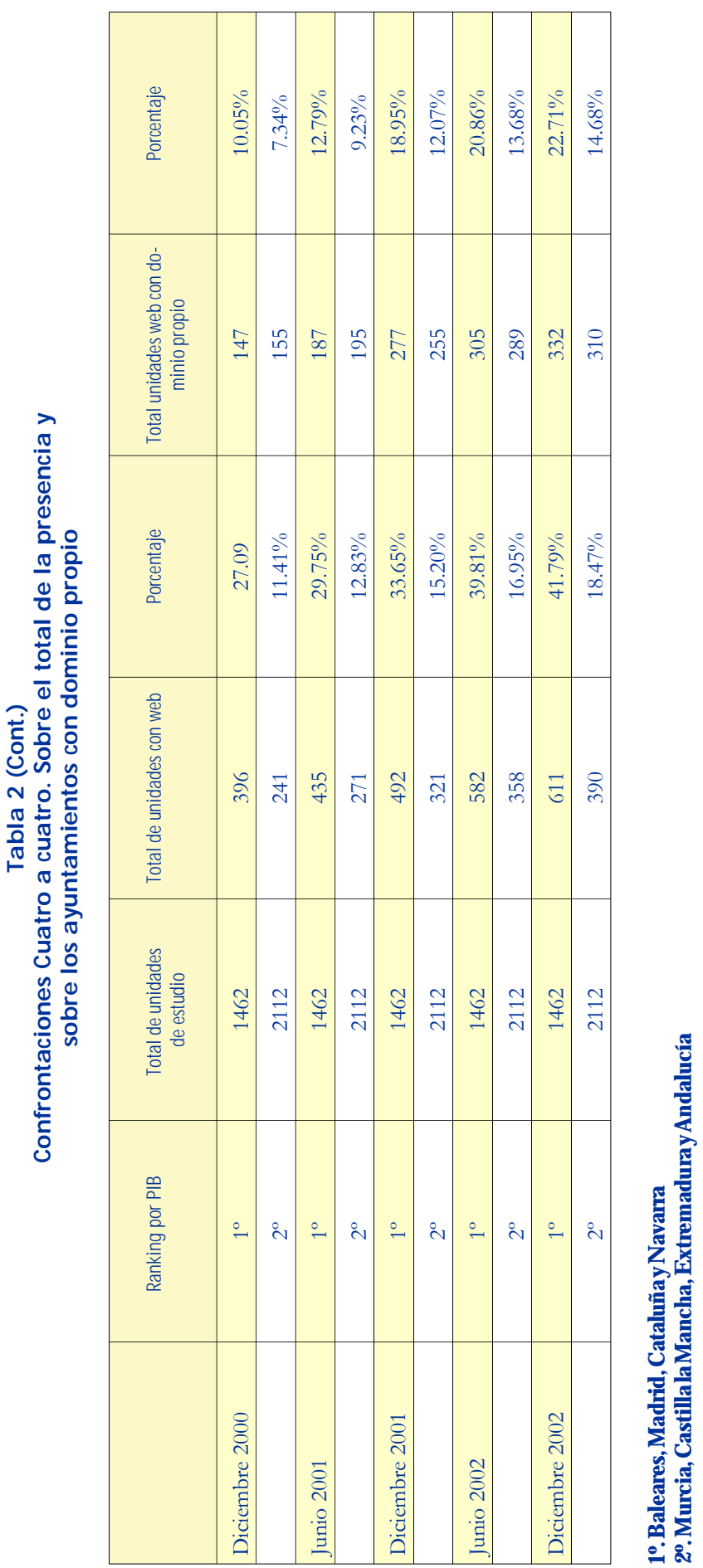




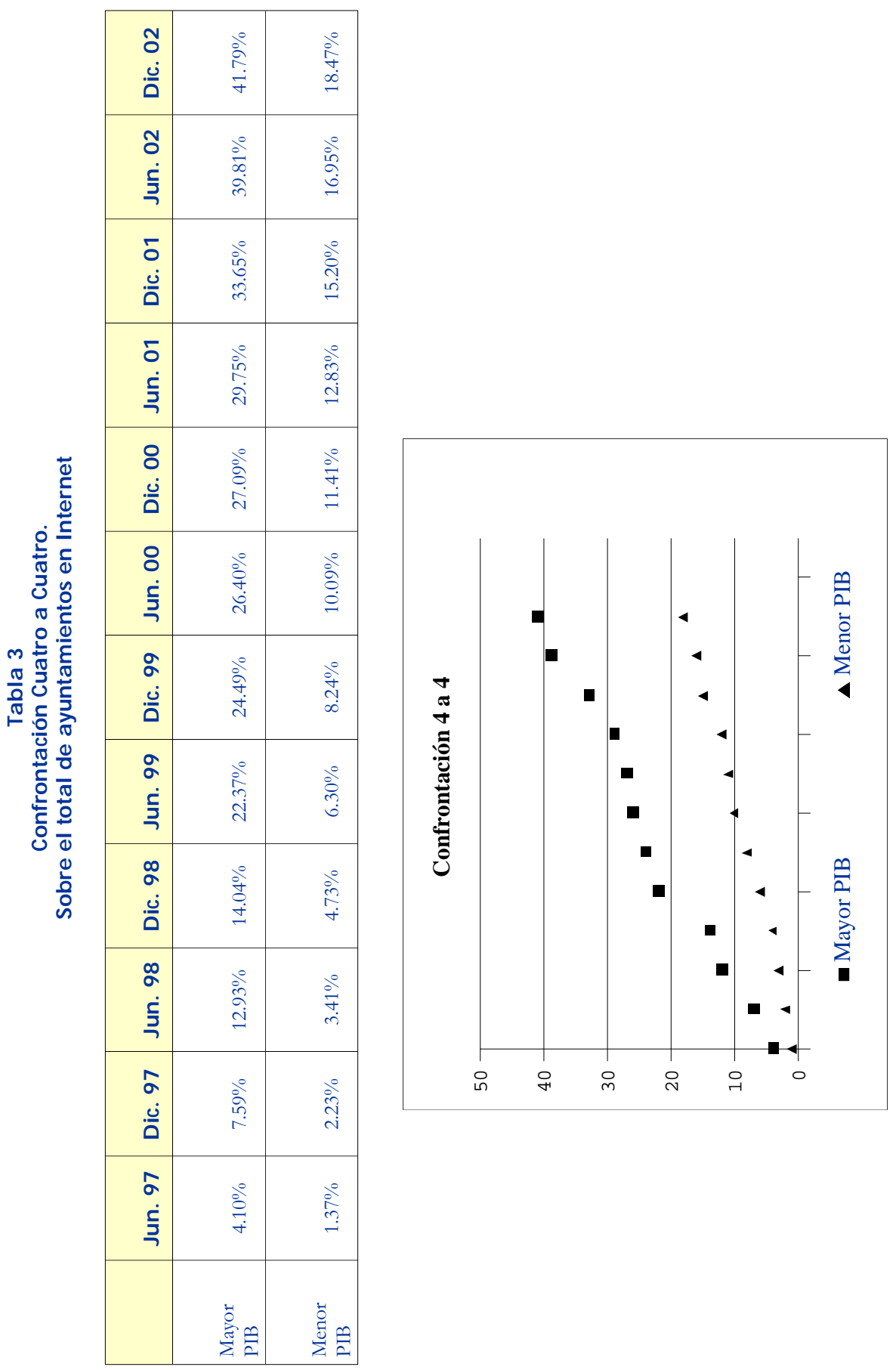




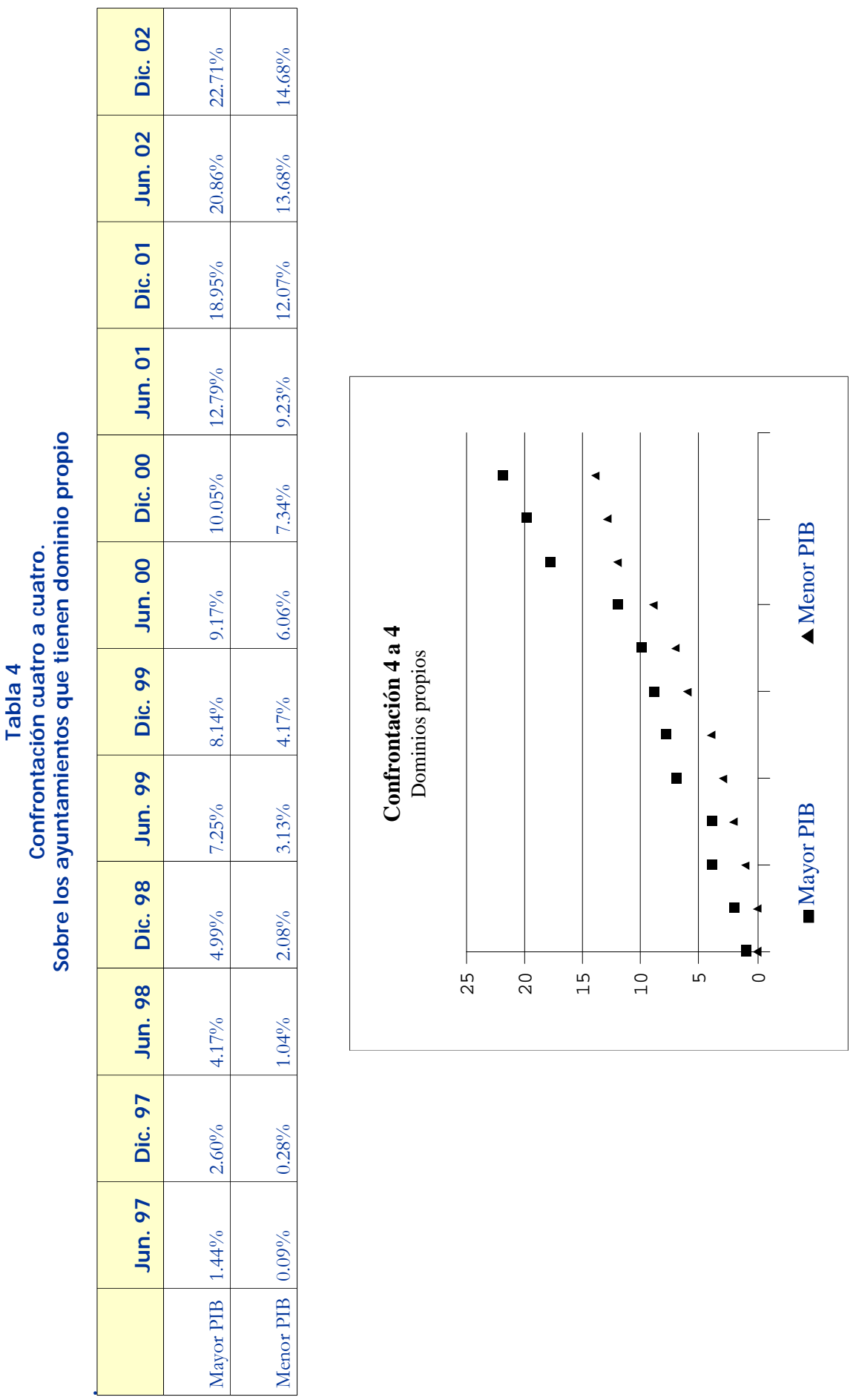




\section{CONCLUSIONES}

* El análisis cuantitativo confirma la evolución clara y constante de la presencia de los ayuntamientos en Internet.

* Una característica propia de la evolución web es que algunas unidades tardan más en incorporarse, pero por lo general la evolución es bastante rápida, y acortan las diferencias con las otras.

* El dominio propio, tal y como se aventuraba en la hipótesis, es un distintivo claro en la evolución de la presencia de los ayuntamientos en la red.

* La agregación de datos entre regiones sirve también para evaluar este desarrollo y compararlo con el resto de los parámetros.

* Hay una diferencia importante de desarrollo entre los gobiernos locales de las 17 CC.AA.; mientras que hay 5 que superan el 50\% de incorporación, existen otras con cifras muy bajas. Pero hay que tener en cuenta el número de municipios sobre el que se realiza el análisis, ya que existe una diferencia muy acusada entre regiones. Así, tal y como se puede comprobar en la tabla1, algunas regiones apenas llegan a los cien municipios, mientras otras tienen cifras muy elevadas, como los casos de Castilla y León con 2247 corporaciones.

* La utilización de la comparativa 4 a 4 sirve para estudiar la evolución de los gobiernos locales de las 17 CC.AA. Los ayuntamientos que pertenecen a comunidades autónomas con mayor PIB son los que mayor desarrollo tienen en cuanto a la incorporación a Internet (tabla 3), y a la evolución hacia dominio propio (tabla 4), aunque en este segundo caso la diferencia es menos acusada.

\section{BiBLIOG RAFÍA}

ABRAMSON, J.R.; Arteton, C. F. y Orren G. O. Thedetroniccommonmealth: Theimpad of newmedia teechndoges on demoratic pditics New York: Basic Books, 1988.

ARTETON, C.F. Tdedemoratics Can tedndogy protet denocaag?. Beverly Hills: Sage, 1987.

ASHBAUGH, Sam. "The Government Performance and Results Acts: Lessons for State and Local Government", en Govemment FinanceRe viens 2001, vol. 17, n 2, p. 19-24.

BARNEY, Darin. PromtheusWired TheHqpefor Demoragin theAgeof Net work Tehndogy. Chicago: University Press, 2000.

BIMBER, B. TheDemographics of Intemet A cess [en línea]. plain http://www.sscf.ucsb.edu/survey1/access.html. [Ultima consulta 1997] (No disponible actualmente en Internet). 
BIMBER, B. TheIntemetandPditical Commuicarionin the1996 Eletrion Set san: rarch Note [en línea]. Disponible en plain http://www.sscf.ucsb.edu/survey1/mobilize.htm. [Última consulta 1997] (No disponible actualmente en Internet).

- -.The Intemet and pditical transformation [en línea]. Disponible en plain http://www.sscf.ucsb.edu/survey1/poltran2.htm. [Última consulta 1996] (No disponible actualmente en Internet).

BIRKINSHAW, Patrick J. "Freedom of Information in the U.K.: A progress Report", en GaemmentInfamationQuartely, 2000, vol. 17, no 4, p. 419-425.

BOURQUARD, J. A. \& Greenberg, P. Savy Citizens... 1995; Rheingold, H. TheV irtual Commmity Homestanding on the detroric Frontier. Reading: Addison-Wesley, 1993.

BOWSER, Brandi. "Opening the window to on-line democracy", en Ame nicanCity \& Canty, 1998, vol. 113, nº 1, p. 32-39.

COLEMAN, J.S. Foundationsofsoial theery. Cambridge: Harvard University Press, 1990.

CORNELLA, A. "Information Policies in Spain", en Gouenment Information Quartely, 1998, vol. 15, nº 2, p. 197-221.

CORNELLA, A. La cultura de la información como institución previa a la Sociedad de la Información. En: Cid, Pilar; Baro Jaume (eds). Anuario SOCADI deDœumentacóneInformadón Barcelona: Societat Catalana de Documentació e Informació, 1998, p. 39-50.

CORNELLA, Alfons. "Políticas de información y rankings de países", en El profesional delainformacón julio-agosto 1996, vol. 7, nº 7-8, p. 24.

COURET, Christina. "Connecting the dots: The changing face of local government", en AmericanCityandCaunty, may 2000, vol. 115, nº, 13 p.

CULLEN, Rowena J. "Does Performance Measurement Improve Organisational Effectiveness: A post Modern Analysis", en Proceedings of the 2nd Northumbria Intemational Confereneon Peformane Meesuremet in Libravies andInformation Sevices held at Longhurst Management and Training Center, Longhurst Hall, Northumberland, England, September 1-6, 1997.

DUTTON, W.H. "Political science research on teledemocracy", en Soial SaiereComputer Review 1992, vol. 10, p. 505-522.

DUTTON, W.H.; Blumler, J. y Kraemer, K (eds). Wiredaties Shapingthefur tureffommmications Boston: Hall, 1987. 
ESPAÑA. "Avances notables en la Administración Pública electrónica", en InfomeEspaña2002, unaintepprezacóndesurølidadsocial. Madrid: Fundación Encuentro, 2002, p. 30-37.

ESPAÑA. Real Decreto 208/1996, de 9 de febrero, sobre Regulación de los Servicios de Información Administrativa y Atención al Ciudadano.

ESPAÑA. Ley Orgánica 15/99 de Protección de Datos Personales.

ESPAÑA. Real Decreto 263/1996, de 16 de febrero, sobre Regulación de la Utilización de técnicas electrónicas, informáticas y telemáticas en la Administración.

ESPAÑA. Decreto 209/2003, de 21 de febrero, por el que se regulan los registros y las notificaciones telemáticas, así como la utilización de medios telemáticos para la sustitución de la aportación de certificados por los ciudadanos.

ESPAÑA. Ley 34/2002, de 11 de julio, de servicios de la sociedad de la información y de comercio electrónico.

ESPAÑA. Real Decreto-ley 14/1999, de 17 de septiembre sobre firma electrónica.

ESPAÑA. Plan de acción InfoXXI [en línea]. Disponible en http://infoxxi.es. [Última consulta 06-10-2003].

ESPAÑA. Red. es [en línea]. Disponible en http://www.red.es [Última consulta 06-10-2003].

ETZIONI, A. Thenewgddennule Communityandmalityinademoraticsociey. New York: Basic Books, 1996.

FAHEY, Tom. DicionariodeIntemet México: Prentice-Hall, 1995.

HAGUE, B. y Loader, B. Digital Demmagy: discaurseanddaision-makinginthe infomationage London: Routledge, 1999.

HERNON, Peter. U.S "Government on the Web: a comparison between the United States and New Zealand", en GouemmentInfomationQuarterly, 1998, vol. $15, n^{\circ} 4$, p. 419-444.

HERNON, P.; Schuler, John A. y Dugan, Robert E. U.SGoumment onthe Wea GettingtheInformationYauNeed Englewood, Co: Libraries Unlimited, 1999.

HORROCKS, I. y Hambley, N. "Developments the 'webbing' of British Local Government”, en PublicMonejandManagement, 1998, vol. 18, nº 2.

LAYNE, Karen y Lee, Jungwoo. "Developing fully functional E-government: a four stages model”, en Govemment Infomation Quartely, 2001, vol. 18, n 2, p. 122-136. 


\section{Investigadón Biblicteedógiav. 18 No. 36 enero/ junio de 2004}

LÓPEZ CAMPS, Jordi y Gadea Carrera, Albert. Serviral dudadana Gestión deCalidadenlaAdministración Pública. Barcelona: Gestión 2000, 1995.

LÓPEZ, J. y Gadea, A. Unanuevaadministracoón pública. Estrategjasymátobs paramigararlaficienciadl eGdiema Oñati: Instituto Vasco de Administración Pública, 2001.

\section{LÓPEZ, J. y Leal, I. E-Gdaiema GdbemarenlaSociedaddd Conoimienta Oña-} ti: Instituto Vasco de Administración Pública, 2002.

Los ayuntamientos se regulan en España por la Ley de Bases de Régimen Local, y por el Reglamento de Organización, Funcionamiento y Régimen Jurídico de las Entidades Locales.

LOZANO, L. "La Sociedad de la Información en Europa: e-Europe", en Seminario "La nueva administración de Estada eAdministración: Conetandb conlos ciudadanos'. 21 y 22 de mayo de 2001. p. 165-173.

MARLIN, A. S. "Politics on the web, why and where?", en Campaigns \& Eletions, 1999, vol. 20, p. 11-12.

McCLURE, C.R.; Wyman, S.K. y Beachboard, J.C. QualityCriteriaforEvaluatingInfomation ResourcesandSevicesAvailablefromFederal WebSites [en línea]. Disponible en

ttp://www.oclc.org/research/publications/art/1997/mcclu-

re/app3.htm. [Última consulta 12-04-2004].

MCGARWEY, J. Residential PC penetration nears 50 percent. Interadive Wedk [en línea]. plain http:/ / connect.colorado.edu/community/resources/harsh/acess.html. [Última consulta 10-3-1998]. (No disponible actualmente en Internet).

MUÑOZ CAÑAVATE, Antonio. "Los recursos informativos en las redes. La información de las Administraciones Públicas españolas en Internet: un estudio sobre su evolución”, en Bdeł́ndeAnabad 2000, abril-junio, $\mathrm{n}^{\circ} 2$, p. $175-190$.

MUÑOZ CAÑAVATE, Antonio. "Un estudio sobre políticas de información en las regiones españolas. El uso del Weben las Administración regional", en DoumentacóndelasCieniasdelasInformacón 2000, n 23, p. 123-139.

MUÑOZ CAÑAVATE, Antonio. “Una aproximación a la información del sector público: la información de las Administraciones Públicas", en RevistaGeneral deInfomaciónyDoumentación 2001, vol. 11, n 1, p. 33-47.

MUÑOZ CAÑAVATE, Antonio y Chaín Navarro, Celia. “The World Wide Web as an information system in Spain's Regional Administrations (1997-2000)", en Gouemment InfomationQuartely. 2004 (en prensa). 
MUÑOZ CAÑAVATE, Antonio y Vivas Moreno, Agustín. “Gestión de la información administrativa de las Administraciones locales españolas en Internet: presencia institucional en la red", en RevistaEspañdadeDoamentacón Científica, 2001, vol. 24, nº 2, p. 162-177.

MUSSO, Juliet; Weare, Chistopher y Hale, Matt. "Designing Web Technologies for Local Governance Reform: Good Management or Good Democracy?”, en Pditical Commmication 2000, vol. 17, p. 1-19.

NEUBAUER, B.J. y Commuri, Ch. "Government Administration in the Internet Age Cyberspace and the Information Elite", en Meding of the AmericanPditical ScienceAssociation 1996, 29 aug-1 sep, San Francisco.

NISTAL, Gloria y Castro, José M. RacionalizacióndelaspdíticasdeAdninis tracón detrónica enlaUniónEuropea [en línea] (Estudio encargado por la Presidencia Española del Consejo de la Unión Europea). 2002. Disponible en

http:// www.csi.map.es/csi/pdf/RacionalEsp.pdf [Última consulta: 28 octubre 2003].

PETER, G.B. Thefutureofgreming Farremegingmodds. Lawrence: University Press of Kansas, 1996.

PUTNAN, R.D. Makingdemmagy wark: CivictraditionsinmodemItaly. Princeton: University Press, 1993

RAAB, C., Bellamy,C., Taylor,J., Dutton, W., y Peltu, M. The information polity: Electronic democracy, privacy and surveillance. En W.H. Dutton (ed.). Infomationandcommunicationtechndoges V isionsandrealities New York: Oxford Universitiy Press, 1996, p. 283-299.

RAMIO, C. El papel de la eadministración ante los retos de las administraciones públicas de América Latina: eadministración y sector público en el siglo de la globalización. VII CongesoIntemacional de CLAD sobrelaRe fomadd EstadbydelaAdministraciónpública [en línea]. Lisboa (Portugal), 8-11 oct. 2002. Disponible en http://www.clad.org.ve/fulltext/0043805.pdf. [Última consulta 0710-2003].

RHEINGOLD, H. TheV irtual CommunityHomestandinganthedetranicFrontier. Reading: Addison-Wesley, 1993.

REYLEA, Harold. C. "Paperwork Reduction Act Reathorization and Government Information Management”, en Goumment Information Quartety, 2000, vol. 17, n 4, p. 367-384.

SAFAI-AMIN, Minoo. "Information Technologies: challenges and opportunities for local governments", en Jaumal of Govemment Inform tion Jul-Aug.2000, vol. 27, n 4, p. 471-479. 
S. D. "Local Government Go on the WWW", en Fonumfor Pdigy Resererch nens Camden: State University of New Jersey, 1996. p. 1-3 y 15.

STOWERS, G.L. "Becoming cyberactive: State and local government on the World Wide Web", en Goumment Infomation Quartery, 1999, vol.. $16, \mathrm{n}^{\mathrm{o}} \cdot 2$, p. 116-125.

Tsagarousianou, R.T, Tambini, D \& Bryan, C. Cyberdemmagy: Te dondogy, atiesand đivicnewarks New York: Routledge, 1998.

UNIÓN EUROPEA. Lainfomaciónde setorpública unrearsodaveparaEuro pa. COM (1998), 585.

UNIÓN EUROPEA. Infomesdarela evduaióndelosserviaiospúblicoseetrónicos dentrodelaUE [en línea]. 25-26 abril 2002. Disponible en http://www.csic.map.es/csi/pdf/eGovEsp_definitivo.pdf [Fecha de consulta 28-10-2003].

VARLEY, P. “Electronic Democracy”, en TehndogyReview1994, vol. 8, p. 42-52.

YANKELOVICH, D. Comingtopudicjudgement: Makingdemoragy work ina comple warld Syracuse: University Press, 1991.

WEATHERMAN, Mark, S. \& Petro, Jim. “Government Services”, en Ohio CPA Jaumal, Jul-Sep.2000, vol. 59, nº 3, p. 89.

Web del Instituto Nacional de Estadística. http://www.ine.es [última consulta 22 sept. 2003]. La variación con respecto al Padrón de 1998 es de $0.46 \%$, poco significativa. En este estudio se exceptuaron Ceuta y Melilla, al ser ciudades autónomas españolas, y no encontrarse enclavadas en ninguna provincia.
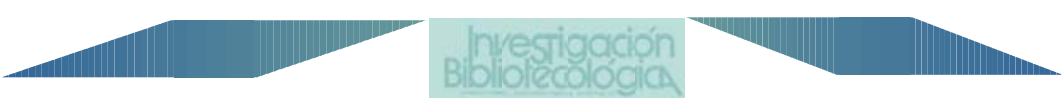